\title{
Helium self-enrichment in globular clusters and the second parameter problem in $\mathrm{M} 3$ and $\mathrm{M} 13$
}

\author{
V. Caloi ${ }^{1}$ and F. D'Antona ${ }^{2}$ \\ ${ }^{1}$ Istituto di Astrofisica Spaziale e Fisica Cosmica, INAF, Roma \\ e-mail: vittoria.caloi@rm.iasf.cnr.it \\ 2 INAF - Osservatorio Astronomico di Roma \\ e-mail: dantona@mporzio.astro.it
}

Received 2 August 2004 / Accepted 3 February 2005

\begin{abstract}
Inspection of the CM diagrams of globular clusters having similar heavy element content shows that the luminosity of the red giant bump relative to the turnoff $\left(\Delta V_{\mathrm{TO}}^{\text {bump }}\right.$ ) differs by more than 0.1 mag between clusters with different horizontal branch morphology. Unfortunately, careful consideration of the data leaves us with only one pair (M3 and M 13) of clusters suitable for a quantitative discussion. For this pair we consider differences in age and helium content as possible causes for the difference in $\Delta V_{\mathrm{TO}}^{\mathrm{bump}}$, and find more convincing support for the latter. A larger helium content in M 13 stars $(Y \sim 0.28$ vs. $Y \sim 0.24$ ) accounts for various CM diagram features, such as the difference in the luminosity level of RR Lyr variables and of the red giant bump with respect to the turnoff luminosity and the horizontal branch morphology. This enhanced helium can be tentatively understood in the framework of self-enrichment by massive asymptotic giant branch stars in the first $\sim 100 \mathrm{Myr}$ of the cluster life. A modest self-enrichment can be present also in M3 and can be the reason for the still unexplained presence of a not negligible number of luminous, Oosterhoff II type RR Lyr variables. The hypothesis that a larger helium content is the second parameter for clusters with very blue horizontal branch morphology could be checked by an accurate set of data for more clusters giving turnoff, RR Lyrs and bump magnitudes within a unique photometry.
\end{abstract}

Key words. Galaxy: globular clusters: general - stars: horizontal-branch - Galaxy: globular clusters: individual: M 3 Galaxy: globular clusters: individual: M 13 - stars: Hertzsprung-Russell (HR) and C-M

\section{Introduction}

The processes resulting in globular clusters (GCs) are complex, given chemical inhomogeneities and peculiarities, difference in radial distribution of the various cluster stellar components, the permanence of the second parameter mystery, etc. (e.g., Kraft 1994; Gratton et al. 2001; Briley et al. 2004; Catelan et al. 2001). In preceding papers (D'Antona et al. 2002; D'Antona \& Caloi 2004) we examined the possibility of self-enrichment in helium as a consequence of star formation episodes of the material ejected by massive asymptotic giant branch (AGB) stars of the first generation. Such material will be enriched in helium and $\mathrm{N}$, possibly in $\mathrm{Na}$ and $\mathrm{Al}$, owing to the second and third phases of dredge-up. The presence of a spread in helium content may help in explaining features such as blue tails and bimodality in horizontal branches (HBs) (see the above quoted papers), the main point being the reduction in the evolving mass, at a given age, with increasing helium content.

The most obvious candidates for the helium enrichment phenomenon are the clusters with an extended HB, populated in regions difficult to reach with a standard mass loss of about $0.2 M_{\odot}$ and a dispersion of $0.02 M_{\odot}$ (e.g., Lee et al. 1994; Catelan et al. 1998). Among these are many of the so-called second parameter clusters, which have an intermediate heavy element content and an HB mainly populated on the blue side of the RR Lyrae region. We shall look for features which may be caused by an enhanced helium content, from a morphological and evolutionary point of view, leaving aside other considerations (formation, dynamics, ...), since our purpose is to explore the possibilities opened by the original helium enhancement hypothesis without the presumption of a complete solution.

\section{The models}

For the purpose of this investigation, we computed isochrones and HB models, which are shown in Figs. 1 and 2. The code used is ATON2.0 and its improvements (Ventura et al. 1998). The heavy element content $Z=0.001$ has been chosen as close to the value observed in the intermediate metal-poor GCs that we shall consider, and in particular, close to the metallicity of M 3 and M 13 (i.e., Sneden et al. 2004). A helium content of 0.24 has been assumed, close to the cosmological value (Izotov \& Thuan 1998; Spergel et al. 2003).

As we see from Fig. 1, the turnoff in isochrones with the same metal content and increasing helium content becomes 


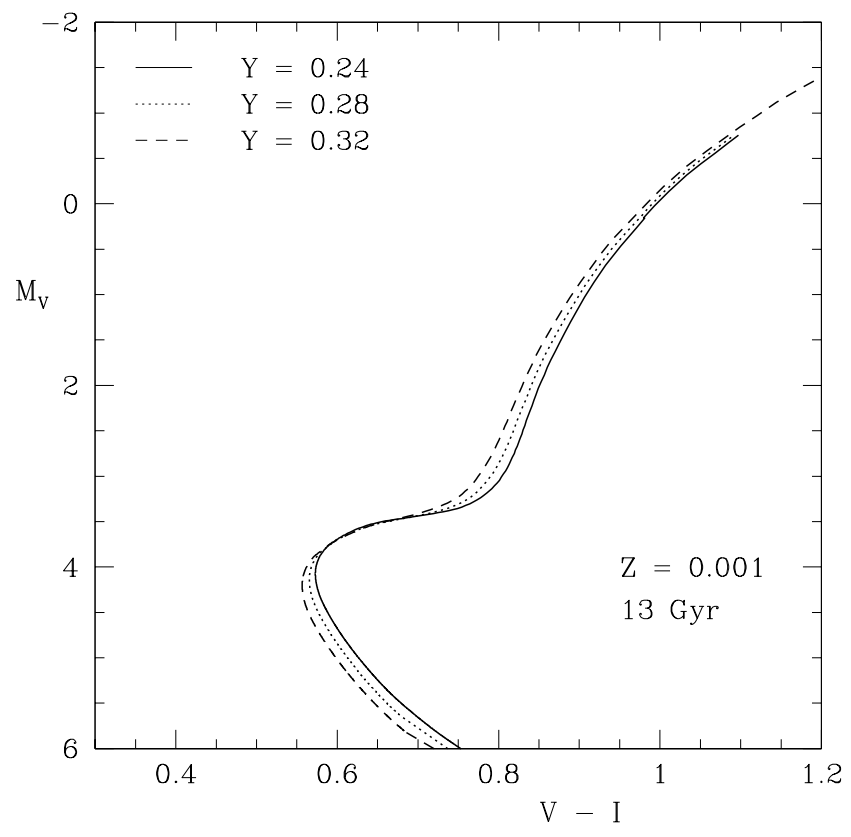

Fig. 1. Isochrones of $13 \mathrm{Gyr}$ for $Z=0.001$ and $Y=0.24,0.28$ and 0.32 (see text).

bluer and fainter. The turnoff at $13 \mathrm{Gyr}$ and $Y=0.28$ is 0.06 mag fainter than with $Y=0.24$, and the shift in colour is of $\sim 0.007 \mathrm{mag}$, both in $(B-V)$ and $(V-I)$. The HB evolutionary tracks with varying $Y$ have been computed with the evolutionary helium core masses $\left(M_{\mathrm{c}}\right.$ in Fig. 2$)$ and surface helium content derived from the first dredge-up ( $Y_{\text {sur }}$ in Fig. 2), and are terminated when the central $Y$ is reduced to 0.10 . The most important feature of the tracks is the increase in luminosity with increasing $Y$ (Sweigart \& Gross 1976): the HB with $Y=0.28$ is on average $\sim 0.20$ mag more luminous than the one with $Y=0.24$.

\section{The bump on the red giant branch}

\subsection{Selection of the sample}

The slowing down of red giant (RG) evolution which takes place when the hydrogen shell reaches the point of the maximum inner expansion of the convective envelope gives origin to the so-called red giant bump (Thomas 1967; Iben 1968); this feature has been observed in many clusters (Ferraro et al. 1999; Zoccali et al. 1999; Riello et al. 2003). We consider intermediate metallicity clusters with very similar heavy element content, and report in Table 1 the magnitude difference between turnoff and RG bump. We considered only clusters for which the data of the turnoff and bump come from the same photometry ${ }^{1}$. Unfortunately, it is not possible to use recent large data sets: i) Rosenberg et al. (1999) give a homogeneous data set for turnoffs and HBs, but not for RG bumps; ii) RG bumps magnitudes are given by Ferraro et al. (1999) (the ones we used),

\footnotetext{
${ }^{1}$ For this reason for NGC 6752 we used the photometry by Buonanno et al. (1986), the only set of data in which the turnoff and RG bump are both identified.
}

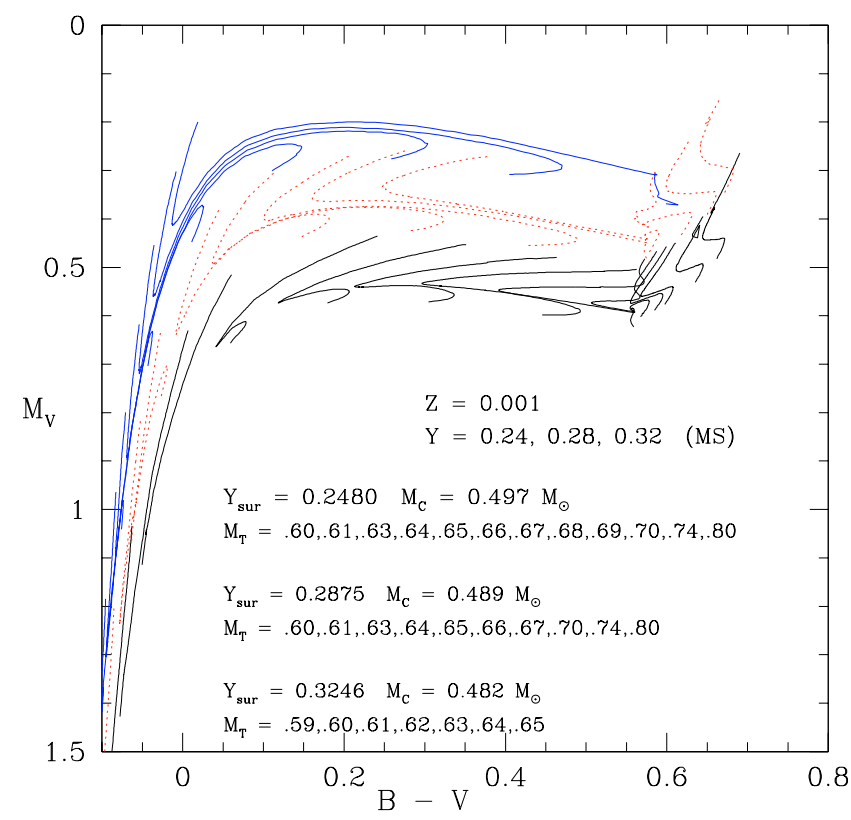

Fig. 2. Theoretical HB evolutionary tracks for $Z=0.001$ and MS helium content $Y=0.24,0.28$ and 0.32 . Total mass $\left(M_{\mathrm{T}}\right)$, helium core mass $\left(M_{\mathrm{C}}\right)$ and surface helium content $\left(Y_{\text {sur }}\right)$ are indicated (see text).

by Zoccali et al. (1999), based on HST observations transformed into the standard $B$ and $V$ system, and by Riello et al. (2003), who leave the data in the HST system. They do so because of possible "deceptive errors in the estimate of the visual magnitudes" introduced by the transformation to the standard Johnson system, which requires knowledge of the cluster reddening. Both Zoccali et al. (1999) and Riello et al. (2003) do not give turnoff estimates.

We used Rosenberg's turnoff magnitudes together with the ones quoted in present paper to reduce the errors, except for NGC 7089 and NGC 6934, not present in Rosenberg's compilation. A meaningful improvement is found only for M3 and M 13, for which the errors in Rosenberg's data are substantially lower than those in Table 1. For the bump magnitudes, the Riello ones are useless for our purpose, and the ones by Zoccali et al. may be used for clusters with low reddening, to reduce the calibration errors mentioned before. We stress that there are no other intermediate metallicity clusters in the literature for which turnoff and bump magnitudes are derived from the same photometry.

\subsection{A hint for differences in $\Delta V_{\mathrm{TO}}^{\text {bump }}$}

In Table 1 we compare the data for clusters with mostly blue HBs with the data for clusters with $\mathrm{HBs}$ well populated in the $B, V$ and $R$ regions. We found only two clusters of this latter type, one of which is the well-studied $\mathrm{M} 3^{2}$.

${ }^{2}$ We did not consider NGC $6229([\mathrm{Fe} / \mathrm{H}]=-1.30)$ for which the bump magnitude is uncertain (Borissova et al. 1997), and NGC 3201 which is 0.1 dex more metal rich than $\mathrm{M} 3([\mathrm{Fe} / \mathrm{H}]=-1.23$, Carretta \& Gratton 1997). 
Table 1. Globular Clusters with very similar heavy element content in the Carretta \& Gratton scale and differing CM diagram features.

\begin{tabular}{|c|c|c|c|c|c|c|c|}
\hline Cluster & Messier & {$[\mathrm{Fe} / \mathrm{H}]_{\mathrm{CG}}$} & $V_{\text {Tо }}$ & $V_{\text {bump }}$ & $\Delta V_{\mathrm{TO}}^{\text {bump }}$ & $V_{\mathrm{RR}}$ & $N_{\mathrm{RR}}$ \\
\hline \multicolumn{8}{|c|}{ Globular Clusters with predominantly blue HB } \\
\hline NGC 1904 & M79 & -1.37 & $19.70 \pm 0.10$ & $15.95 \pm 0.10$ & $3.75 \pm 0.11$ & & \\
\hline NGC 6093 & M 80 & -1.41 & $19.75 \pm 0.10$ & $15.95 \pm 0.10$ & $3.80 \pm 0.14$ & 15.98 & 3 \\
\hline NGC 6205 & M 13 & -1.39 & $18.55 \pm 0.10$ & $14.75 \pm 0.05$ & $3.80 \pm 0.11$ & $14.83 \pm 0.02$ & 7 \\
\hline NGC 6752 & & -1.42 & $17.40 \pm 0.10$ & $13.65 \pm 0.05$ & $3.75 \pm 0.11$ & & \\
\hline NGC 7089 & M2 & -1.38 & $19.60 \pm 0.10$ & $15.85 \pm 0.05$ & $3.75 \pm 0.11$ & $15.926 \pm 0.021$ & 11 \\
\hline \multicolumn{8}{|c|}{ Globular Clusters with $\mathrm{HB}$ populated in the $B, V$ and $R$ regions } \\
\hline NGC 5272 & M 3 & -1.34 & $19.10 \pm 0.10$ & $15.45 \pm 0.05$ & $3.65 \pm 0.11$ & $15.665 \pm 0.013$ & 35 \\
\hline NGC 6934 & & -1.30 & $20.40 \pm 0.15$ & $16.78 \pm 0.10$ & $3.62 \pm 0.18$ & $16.873 \pm 0.017$ & 24 \\
\hline
\end{tabular}

Bibl. CM diagram: M 79: Kravtsov et al. (1997); M 80: Brocato et al. (1998); M 13: Paltrinieri et al. (1998); NGC 6752: Buonanno et al. (1986) as in Ferraro et al. (1999); M 2, M3: Lee \& Carney (1999b); NGC 6934: Piotto et al. (1999).

Bibl. RR Lyr: M 80: Wehlau et al. (1990), the 3 RR Lyr with the best photometry; M 13: Kopacki et al. (2003); M 2: Lee \& Carney (1999a), 11 RRab with stable light curves; M 3: Carretta et al. (1998), 35 RRab with stable light curves chosen by Lee \& Carney (1999b); NGC 6934: Kaluzny et al. (2001), 24 RRab with stable light curves.

Table 2. Additional data for M 3 and M 13 from Rosenberg et al. and Zoccali et al., plus the weighted means with relative errors when used together with data in Table 1.

\begin{tabular}{cccccccc}
\hline \hline Cluster & Messier & $V_{\text {TO }}(\mathrm{R})$ & $V_{\text {bump }}(\mathrm{Z})$ & $V_{\mathrm{TO}}($ mean $)$ & $V_{\text {bump }}($ mean $)$ & $\Delta V_{\mathrm{TO}}^{\text {bump }}$ & $\Delta V_{\mathrm{TO}}^{\text {RR }}$ \\
\hline NGC 6205 & M 13 & $18.50 \pm 0.06$ & $14.70 \pm 0.04$ & $18.51 \pm 0.05$ & $14.72 \pm 0.03$ & $3.79 \pm 0.06$ & $3.68 \pm 0.05$ \\
NGC 5272 & M 3 & $19.10 \pm 0.04$ & - & $19.10 \pm 0.04$ & $15.45 \pm 0.05$ & $3.65 \pm 0.06$ & $3.44 \pm 0.04$ \\
\hline
\end{tabular}

We notice that the the average $\Delta V_{\mathrm{TO}}^{\text {bump }}$ for the blue HB clusters is always larger than for the M3-type clusters: for the former ones we have an average of $3.77 \pm 0.07 \mathrm{mag}$, while for the latter clusters we have $3.64 \pm 0.11 \mathrm{mag}$. To be precise, we should correct this latter value for the difference in average metallicity between the two groups $(-1.39$ vs. -1.32$)$, since both turnoff and bump magnitudes increase with metallicity: this small correction would move the M3-like average to $3.67 \mathrm{mag}$. However, we do not enter in the details of the procedure, because an inspection of the data in Table 1 shows that the error on the difference in $\Delta V_{\mathrm{TO}}^{\text {bump }}$ between the two groups is of the same order of magnitude of the difference itself. So we limit the discussion to the clusters M 3 and M 13, for which a substantial reduction of the errors is possible, as mentioned before. In dealing with these two clusters, we consider them of the same metallicity (see, e.g., Rosenberg et al. 1999).

\subsection{The case of $M 3$ and $M 13$}

In Table 2 we give the available data for M 3 and M 13 from Rosenberg et al. (1999) and Zoccali et al. (1999), the weighted means between these values and the data in Table 1, and the resulting estimate for $\Delta V_{\mathrm{TO}}^{\text {bump }}$. The difference in $\Delta V_{\mathrm{TO}}^{\text {bump }}$ between the two clusters is $0.14 \pm 0.09$ mag. Even if this result is meaningful only at a one $\sigma$ level, the fact that in all blue clusters for which a consistent measure was possible $\Delta V_{\mathrm{TO}}^{\text {bump }}$ was larger than in M3-type clusters appears intriguing enough to be worth further investigation.

\section{Possible causes of the difference in $\Delta V_{\mathrm{TO}}^{\text {bump: }}$ Age or helium difference?}

The main candidates for an influence on the CM diagram features are age and helium content, since in the present case differences in heavy element content are excluded. Age influences $\Delta V_{\mathrm{TO}}^{\text {bump }}$ because the turnoff and the bump fade at a different rate. For the metallicity of these clusters $(Z \sim 0.001)$ and for ages of $12-15 \mathrm{Gyr}$, the fading of the turnoff with age is of about $0.075 \mathrm{mag} / \mathrm{Gyr}$ (present models, D’Antona et al. 1997; Cassisi et al. 1999). For the bump magnitude we have a fading of 0.035-0.04 mag/Gyr (Ferraro et al. 1999; Cho \& Lee 2002, and our own models). So for each Gyr of difference, $\Delta V_{\mathrm{TO}}^{\text {bump }}$ would increase by about $0.04 \mathrm{mag}$.

The difference of $0.14 \pm 0.09$ mag would correspond to an age difference of $3.5 \pm 2.2$ Gyr. A similar estimate of 3 Gyr is often obtained from the difference in HB morphology for the age interval 12-15 Gyr (see, f.e., the discussion in Johnson \& Bolte 1998). For lower ages - M 3 of about $10 \mathrm{Gyr}-$ the age difference becomes of the order of 2 Gyr (Rey et al. 2001).

Let us now consider the effects of an increase in the helium content in M 13. In clusters with $Y \sim 0.28$ the turnoff luminosity would be fainter by about 0.06 mag (our models, see Fig. 1 and D'Antona et al. 2002), while the luminosity of the RG bump would increase by $\sim 0.08-0.09 \mathrm{mag}$, according to our models, and of 0.09 mag according to Riello et al. (2003) ${ }^{3}$. So $\Delta V_{\mathrm{TO}}^{\text {bump }}=0.14 \pm 0.09$ would be given by a helium variation $\Delta Y=0.04+0.02 /-0.03$.

\footnotetext{
${ }^{3}$ We assumed $\Delta M_{\mathrm{bol}} \sim 1.21 \Delta M_{\mathrm{V}}$ as in Cho \& Lee (2002).
} 


\subsection{The role of $R R$ Lyrae variables}

We consider now the characteristics of the RR Lyrae variables in the two clusters. M 3 has a population of almost $200 \mathrm{RR}$ Lyr variables, with a large majority of RRab-type; the average periods are of $0.56 \mathrm{~d}$ and of $0.32 \mathrm{~d}$, for RRab and RRc, respectively (see, e.g., Jurcsik et al. 2003; Clement et al. 2001). In M 13 only 9 RR Lyr stars have been identified, out of which only one is an RRab; the average period of 7 well observed RRc stars is $0.36 \mathrm{~d}$ (Kopacki et al. 2003). Both the average periods and the percentage of RRc stars indicate that variables in M3 belong to the Oosterhoff type I (a well established fact), while those in M 13 more likely belong to the Oosterhoff type II.

Carretta et al. (1998) and Lee \& Carney (1999a,b) link their RR Lyr photometries for M 3 to that of the non-variable stars (Ferraro et al. 1997a), obtaining the same average magnitude of $15.66 \mathrm{mag}$. The same value is found independently by Jurcsik et al. (2003). So for this cluster we have unique photometry for all the data in Table 1.

For M 13 the photometry of RR Lyr stars is different from that of the CM diagram. The RR Lyr magnitudes are given by Kopacki et al. (2003), who also give mean values for some bright red giant variables. For these stars, the average of their mean magnitudes differs by $\sim 0.04$ mag from the average obtained from the photometry given by Pilachowski et al. (1996) for the same objects ${ }^{4}$. This indicates that the two photometries should be consistent within 0.04 mag.

The difference in Oosterhoff type between M 3 and M 13 is part of the well-known second parameter problem: GCs with the same heavy element content and very different HB populations. From Table 2 we see that the luminosity difference between turnoff and RR Lyraes $\Delta V_{\mathrm{TO}}^{\mathrm{RR}}$ is much larger in M 13 than in M 3: $3.68 \pm 0.05$ vs. $3.44 \pm 0.04$ mag (Table 2 ), so that the difference in $\Delta V_{\mathrm{TO}}^{\mathrm{RR}}$ between the two clusters is $0.24 \pm 0.06 \mathrm{mag}$. In the literature, such a difference is generally interpreted as due to a higher luminosity of the blue and RR Lyr regions in M 13; these stars are supposed to be in a more advanced, and more luminous, evolutionary phase than the ZAHB and its vicinity, where most of RR Lyraes in M3 are found. This behaviour would follow from the higher age of M 13: the smaller HB masses would populate the ZAHB only on the blue side of the RR Lyr variables.

\subsection{The case of a difference in age}

If the difference of $0.14 \pm 0.09$ mag between $\Delta V_{\text {TO }}^{\text {bump }}$ in M 3 and M 13 (Table 2) is due to an age difference, M 13 should be older by $3.5 \pm 2.2$ Gyr. In this case, the turnoff in M 13 should be fainter than in M 3 by $\sim 0.075 \mathrm{mag} / \mathrm{Gyr} \times 3.5 \mathrm{Gyr}=0.26 \mathrm{mag}$. However, available observations are in strong disagreement with such a large difference in turnoff luminosity (e.g., Ferraro et al. 1997b; Johnson \& Bolte 1998; Rey et al. 2001). Also, the RR Lyr luminosity level in M 13, following the observed $\Delta V_{\mathrm{TO}}^{\mathrm{RR}}$ (Table 2), would be 0.02 mag fainter than in $\mathrm{M} 3$, and the

\footnotetext{
${ }^{4}$ The variables V11, V15, V17, V18, V19 and V24 have been considered.
}

apparent Oo II type of the RR Lyr variables in M 13 would remain unexplained.

Only considering the minimum difference in age required by the observed difference in $\Delta V_{\mathrm{TO}}^{\text {bump }}$ taking into account the estimated errors (see above), that is $1.3 \mathrm{Gyr}$, we obtain a consistent scenario: the turnoff in M 13 would be fainter by $0.10 \mathrm{mag}$ than the turnoff in M3, and the RR Lyrs in M 13 would be 0.14 mag brighter than in M3. Still, such a small difference in age could lead to the observed difference in HB morphology only for very low ages, 10 Gyr for M 3 (Rey et al. 2001).

\subsection{The case of a difference in helium}

If the age is the same and helium in M 13 is enhanced up to $Y=0.28$, the turnoff in M 13 would be fainter by 0.06 mag and the HB would be brighter by 0.20 mag (see Figs. 1 and 2). This explanation is consistent with the observed difference in $\Delta V_{\mathrm{TO}}^{\mathrm{RR}}$ between M 13 and M3 $(0.24 \pm 0.06 \mathrm{mag})$. In addition, it predicts for the RR Lyraes in M 13 a larger luminosity (by $\sim 0.20 \mathrm{mag}$ ) than for those in $\mathrm{M} 3^{5}$.

Thus, the hypothesis of a difference in the helium content of the stars in these two clusters is the more appealing one, if the $\Delta V_{\mathrm{TO}}^{\text {bump }}$ is confirmed to the level used in this work. Therefore, it would be extremely important to have homogenous sets of data for the turnoff and bump. We could perform this kind of analysis only for the famous pair M 3-M 13.

\section{The detailed fitting of M 3 and $M 13$ with differing helium contents}

Beside the relative luminosity levels, we have to check whether the (subtle) differences in the CM diagram introduced by a helium increase are supported by observations. In Fig. 3a we show the fit of the fiducial lines for M3 given by Johnson \& Bolte (1998) with the isochrone of $13 \mathrm{Gyr}, Z=0.001$, $Y=0.24$, and the relative HB models. Similarly, in Fig. 3b the fit is shown for M 13, but with an isochrone corresponding to $Y=0.28$; the average position of six RR Lyr variables from Kopacki et al. (2003) is also indicated. Different values for $Y$ not only provide a better explanation of the relative luminosities of the turnoff, HB and bump, but also a better morphological fit. To further stress this point, we show in Fig. 3c a fit of the M 13 fiducial sequence with isochrones with $Y=0.24$ and ages of 13 and 14 Gyr: there is an evident difficulty in obtaining a satisfactory fit, in contrast with the increased helium case.

The HB stellar distribution (second parameter problem) can be explained. In D'Antona et al. (2002) we examined the consequences on the HB population of an increase in $Y$ from 0.24 to 0.28 . To complete the discussion, we summarize the main effects. In M 3, assuming $Y=0.24$ on the main sequence, we have that the bulk of the HB population covers the mass interval $0.60-0.70 M_{\odot}$, with the peak at the RR Lyr region; a sparse tail toward the blue would require lower masses. Since the evolving giant (in the absence of mass loss) at the helium flash is of about $0.82 M_{\odot}$, the implied mass loss is $0.17 \pm 0.05 M_{\odot}$.

\footnotetext{
5 To be precise, the RR Lyrs become brighter by 0.18 mag.
} 

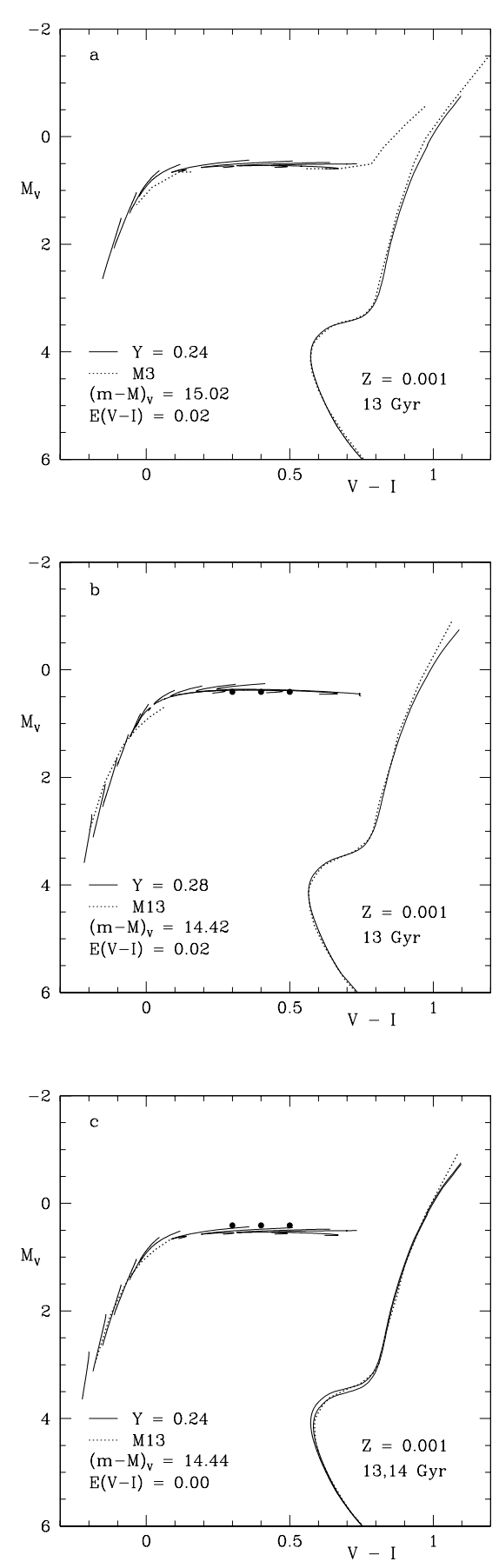

Fig. 3. a) CM diagram of M 3 (data by Johnson \& Bolte) compared with an isochrone of $13 \mathrm{Gyr}, Y=0.24$ and relative HB tracks. b) CM diagram of M13 (data by Johnson \& Bolte, RR Lyr level from Kopacki et al., full dots) compared with an isochrone of $13 \mathrm{Gyr}$, $Y=0.28$ and relative HB tracks. c) CM diagram of M 13 (data by Johnson \& Bolte) compared with isochrones of 13 and 14 Gyr and relative HB tracks for $Y=0.24$. The $Y=0.28$ models give a better morphological fit of the turnoff region of M 13.

With a helium content of 0.28 and 13 Gyr of age, the evolving giant at the helium flash is of $0.766 M_{\odot}(Z=0.001$, as before). With the same mass loss as in M3, the maximum HB mass turns out $0.646 M_{\odot}$, in the middle of the RR Lyr region (see Fig. 2), while the minimum mass is of about 0.546 , almost down at the level of the turnoff. If mass loss along the giant branch increases with decreasing mass (e.g., Lee et al. 1994), lower masses can be reached, but see also Lee \& Carney (1999b). So M 13 would naturally shift its HB population according to observations. We have been using a helium content of 0.28 for simplicity, while, most likely, a spread in $Y$ is to be expected, starting from $\sim 0.28$ and reaching well beyond 0.30 (D'Antona \& Caloi 2004). We elaborate briefly on the subject of self-enrichment in the following section.

\section{The self-enrichment scenario}

In a preceding paper (D'Antona et al. 2002) we considered helium enrichment as mostly related to the origin of very blue HB tails in second parameter clusters such as M13 and NGC 6752. We are now proposing that the overall difference between M 3 and M 13 CM diagram morphologies is due to the different helium content. Thus all stars in M 13 should have an enriched helium content with respect to the Big Bang abundance. How is this possible? We suggest that M 13 represents an extreme case of self-enrichment. Among GCs which show abundance spreads, we have identified NGC 2808 as a cluster in which at present half of the stellar population has a normal $Y$, and the other half is helium enriched at various degrees (D'Antona \& Caloi 2004). The normal $Y$ population is responsible for the red part of the $\mathrm{HB}$, and the enriched one is responsible for the blue side of the $\mathrm{HB}$ and for the blue tails. We have shown that this may happen only if the number of low mass stars with normal $Y$ content present in the cluster is much smaller than expected on the basis of an initial mass function which is, on the one side not too implausible, and on the other, able to explain the blue HB stars as born from the helium enriched ejecta of the massive AGBs. Then many low mass stars of the first generation must have been lost by the cluster, perhaps because the intermediate mass stars were much more concentrated into the central regions (D'Antona \& Caloi 2004). In M 13 there is no red HB clump: in this case, the first generation stars must have been completely lost by the cluster. This hypothesis is certainly difficult to accept without detailed modeling. M 3 and M 13 are very similar in any other respect: present day mass, central density, relaxation time. However, this kind of scenario is still at its early stages. Notice in addition that Salaris et al. (2004), in a detailed analysis of the $R$ parameter in GCs, detect a significant spread of helium content towards higher abundances, in the clusters having very blue HBs. This is a further indication in favour of our hypothesis.

Beside the difficulties with the cluster dynamics, there is the further difficulty that, in this scheme, all M 13 stars should be helium enriched, while the observations show that not all of them are oxygen poor (e.g., Sneden et al. 2004), a feature that is considered to accompany processed matter. However, it has been shown that, while most of the AGB matter from which the second generation stars are born is helium enriched due to the action of the second dredge-up, not necessarily all this matter is oxygen-depleted (Ventura et al. 2001, 2002).

Is M 3 devoid of self-enrichment? This may not be the case, as suggested by the presence of luminous RR Lyrae variables. 


\subsection{The case of the luminous $R R$ Lyrae variables in $M 3$}

Recently it has been noticed that a few RR Lyr variables in M 3, the prototype of the Oo I type clusters, show Oo II characteristics (Clement \& Sheldon 1999; Corwin \& Carney 2001, who quote similar observations by Belserene 1954). Jurcsik et al. (2003) made a detailed study of about 150 RR Lyr stars (of both types ab and c), and found that they could be classified into four groups, according to their mean magnitudes and periods. The most luminous sample statistically has Oo II properties regarding the mean periods and $R_{a b} / R_{c}$ number ratio. They found also that the various samples can be identified with different stages of HB evolution, with some difficulty for the most luminous one. This sample has a luminosity at which evolution is rather fast (the last phases of central helium burning), too fast to support the existence of more than $20 \%$ of the observed RR Lyr stars. As Jurcsik et al. (2003) remark, the discrepancy could be removed by the presence of the (infamous) "breathing pulses", that is, of a final phase of helium mixing in convective cores, when helium abundance is approaching zero (e.g., Castellani et al. 1985; Dorman \& Rood 1993; Caloi \& Mazzitelli 1993), but at present the phenomenon is not considered real and $\mathrm{HB}$ evolution is computed ignoring it.

Keeping in mind such a possibility, we can consider other solutions to the problem of Oo II RR Lyrs in M 3. Considering multiple star generations with helium enrichment, we can hypothise that this cluster also presents a certain amount of second generation members formed from the helium-rich ejecta of AGB stars. Other than the luminous RR Lyrs, other features could be interpreted in terms of variable helium content: i) the large range in period covered by $\mathrm{RR}_{\mathrm{d}}$ variables (Clementini et al. 2004); ii) the great length of the HB, since the lower mass of helium-rich red giants, at a given age, helps to generate very blue HB stars hotter than the main body of $\mathrm{HB}$ population (Ferraro et al. 1997a, Fig. 18); the spread in luminosity and colour in the subgiant region (as noted by Clementini et al., see Fig. 4 in Corwin \& Carney 2001; and Fig. 15 in Ferraro et al. 1997a). In this respect, we notice that, according to recent estimates (Sneden et al. 2004), about one-third of the observed giants in M 3 are oxygen poor. In the hypothesis mentioned before (conversion of oxygen to nitrogen in the envelopes of AGB stars of a preceding generation), these stars could produce the luminous RR Lyr variables, also being helium enriched.

\section{Discussion}

The impressive amount of detailed information recently acquired on the chemical composition of GC members down to the main sequence has profoundly changed our perception of these most ancient stellar systems. Complex processes, both chemical and dynamical, must have taken place in their formation phase, leaving characteristic marks on the stellar chemistry and population.

Here we have tried to relate some photometric features in $\mathrm{CM}$ diagrams to chemical peculiarities, such as helium enrichment. Johnson \& Bolte (1998) suggested a higher helium content in M13 as the best explanation for the
CM diagram morphology differences between this cluster and M3, and widely discussed the subject. Besides the turnoff shape (see Fig. 3), we consider the luminosity of the RG bump with respect to the turnoff, and find that it correlates with the HB type: the metal content being the same, clusters with a blue HB have a larger $\Delta V_{\mathrm{TO}}^{\text {bump }}$ than clusters with a uniformly populated $\mathrm{HB}$.

Considering in detail M3 and M 13, we examine both the difference in age and in helium content as possible causes of this feature, and find that the latter is the more plausible, if one considers the luminosity level of the RR Lyr variables. The presence of Oo II type RR Lyrs in M3 also can be interpreted in terms of a helium-enriched stellar component.

The difference in helium content between M 3 and M 13 appears as a possible "second parameter", at least with regard to this pair of clusters. The difficulties are various, mainly arising from our ignorance of the GC formation phases and of the initial mass function(s). With respect to similar suggestions in the past, the hypothesis presented is supported by detailed investigations on AGB ejecta composition and by the recognized necessity for a primordial contamination of main sequence stars in many clusters, as well as by the interpretation of CM features otherwise not easily understood (HB and bump luminosities). Partial pollution does not help explaining the origin of very blue HB structures (Caloi 2001; D’Antona et al. 2002), while an increased structural helium content does (D'Antona et al. 2002). An important contribution to the problem would be to check the presence of chemical peculiarities in the luminous, OoII-type RR Lyr variables in M3, that we proposed as candidates for second generation, helium-increased structures.

\section{References}

Belserene, E. P. 1954, AJ, 59, 406

Borissova, J., Catelan, M., Spassova, N., \& Sweigart, A. V. 1997, AJ, 113,692

Briley, M. M., Harbeck, D., Smith, G. H., \& Grebel, E. K. 2004, AJ, 127, 1588

Brocato, E., Castellani, V., Scotti, G. A., et al. 1998, A\&A, 335, 929

Buonanno, R., Caloi, V., Castellani, V., et al. 1986, A\&AS, 66, 79

Caloi, V. 2001, A\&A, 366, 91

Caloi, V., \& Mazzitelli, I. 1993, A\&A, 271, 139

Carretta, E., \& Gratton, R. G. 1997, A\&AS, 121, 95

Carretta, E., Cacciari, C., Ferraro, F. R., Fusi Pecci, F., \& Tessicini, G. 1998, MNRAS, 298, 1005

Cassisi, S., Castellani, V., Degl'Innocenti, S., Salaris, M., \& Weiss, A. 1999, A\&AS, 134, 103

Castellani, V., Chieffi, A., Tornambè, A., \& Pulone, L. 1985, ApJ, 296, 204

Catelan, M., Borissova, J., Sweigart, A. V., \& Spassova, N. 1998, ApJ, 494, 265

Catelan, M., Ferraro, F. R., \& Rood, R. T. 2001, ApJ, 560, 970

Cho, D.-H., \& Lee, S.-G. 2002, AJ, 124, 988

Clement, C. M., \& Shelton, I. 1999, ApJ, 515, L85

Clement, C. M., Muzzin, A., Duffon, Q., et al. 2001, AJ, 122, 2587

Clementini, G., Corwin, T. M., Carney, B. W., \& Sumerel, A. N. 2004, AJ, 127, 938

Corwin, T. M., \& Carney, B. W. 2001, AJ, 122, 3183

D’Antona, F., \& Caloi, V. 2004, ApJ, 611, 871 
D’Antona, F., Caloi, V., \& Mazzitelli, I. 1997, ApJ, 477, 519

D'Antona, F., Caloi, V., Montalban, J., Ventura, P., \& Gratton, R. 2002, A\&A, 395, 69

Dorman, B., \& Rood, R. T. 1993, ApJ, 409, 387

Ferraro, F. R., Carretta, E., Corsi, C. E., et al. 1997a, A\&A, 320, 757

Ferraro, F. R., Paltrinieri, B., Fusi Pecci, F., et al. 1997b, ApJ, 484, L145

Ferraro, F. R., Messineo, M., Fusi Pecci, F., et al. 1999, AJ, 118, 1738

Gratton, R. G., Bonifacio, P., Bragaglia, A., et al. 2001, A\&A, 369, 87

Iben, I. Jr. 1968, Nature, 220, 143

Izotov, Y. I., \& Thuan, T. X. 1998, ApJ, 500, 188

Johnson, J. A., \& Bolte, M. 1998, AJ, 115, 693

Jurcsik, J., Benko, M., Bakos, G. A., Szeidl, B., \& Szabo, R. 2003, ApJ, 597, L49

Kaluzny, J., Olech, A., \& Stanek, K. Z. 2001, AJ, 121, 1533

Kopacki, G., Kolaczkowski, Z., \& Pigulski, A. 2003, A\&A, 398, 541

Kraft, R. P. 1994, PASP, 106, 553

Kravtsov, V., Ivanov, A., Samus, N., et al. 1997, A\&AS, 125, 1

Lee, J.-W., \& Carney, B. W. 1999a, AJ, 117, 2868

Lee, J.-W., \& Carney, B. W. 1999b, AJ, 118, 1373

Lee, Y.-W., Demarque, P., \& Zinn, R. 1994, ApJ, 423, 248

Paltrinieri, B., Ferraro, F. R., Fusi Pecci, F., \& Carretta, E. 1998, MNRAS, 293, 434
Pilachowski, C. A., Sneden, C., Kraft, R. P., \& Langer, G. E. 1996, AJ, 112, 545

Piotto, G., Zoccali, M., King, I. R., et al. 1999, AJ, 117, 264

Rey, S. C., Yoon, S. J., Lee, Y. W., Chaboyer, B., \& Sarajedini, A. 2001, AJ, 122, 3219

Riello, M., Cassisi, S., Piotto, G., et al. 2003, 410, 553

Rosenberg, A., Saviane, I., Piotto, G., \& Aparicio, A. 1999, AJ, 118, 2306

Salaris, M., Riello, M., Cassisi, S., \& Piotto, G. 2004, A\&A, 420, 911

Sneden, C., Kraft, R. P., Guhathakurta, P., Peterson, R., \& Fulbright, J. P. 2004, AJ, 127, 2162

Spergel, D. N., Verde, L., Peiris, H. V., et al. 2003, ApJS, 148, 175

Sweigart, A. V., \& Gross, P. G. 1976, ApJS, 32, 367

Thomas, H. C. 1967, Z. Astrophys., 67, 420

Ventura, P., Zeppieri, A., Mazzitelli, I., \& D’Antona, F. 1998, A\&A, 334,953

Ventura, P., D’Antona, F., Mazzitelli, I., \& Gratton, R. 2001, ApJ, 550, L65

Ventura, P., D’Antona, F., \& Mazzitelli, I. 2002, A\&A, 393, 215

Wehlau, A., Sawyer Hogg, H., \& Butterworth, S. 1990, AJ, 99, 1159

Zoccali, M., Cassisi, S., Piotto, G., Bono, G., \& Salaris, M. 1999, ApJ, 518, L49 